\title{
Preliminary Evaluation of Diagnostic Tests for Avian Influenza Using the Markov Chain Monte Carlo (MCMC) Method in an Emergency Surveillance
}

\author{
Takehisa YAMAMOTO ${ }^{1)}$, Toshiyuki TSUTSUI ${ }^{1)}$, Akiko NISHIGUCHI ${ }^{1)}$, Sota KOBAYASHI ${ }^{1}$, Kenji TSUKAMOTO $^{2)}$, \\ Takehiko SAITO ${ }^{2)}$, Masaji MASE ${ }^{2)}$ and Masatoshi OKAMATSU' \\ ${ }^{1)}$ Epidemiological Research Team and ${ }^{2)}$ Research Team for Zoonotic Diseases, National Institute of Animal Health, 3-1-5 Kannondai, \\ Tsukuba-shi, Ibaraki-ken 305-0856, Japan
}

(Received 2 August 2006/Accepted 22 February 2007)

ABSTRACT. In June 2005, an outbreak of avian influenza (AI) caused by a low pathogenic H5N2 virus was identified in Japan. A serological surveillance was conducted because the infected chickens did not show any clinical signs. The Markov Chain Monte Carlo Method was used to evaluate the performances of serological HI and AGP tests because there was not enough time when the surveillance was initiated to conduct a test evaluation. The sensitivity of the AGP test (0.67) was lower than that of the HI test (0.99), while the specificities were high for both tests ( 0.96 for AGP and 0.90 for HI). Based on the low sensitivity of the AGP test, the HI test was used for primary screening in later stages of the epidemic.

KEY WORDS: avian influenza, diagnostic tests, Markov Chain Monte Carlo method.

J. Vet. Med. Sci. 69(6): 673-675, 2007

An outbreak of avian influenza (AI) caused by a low pathogenic H5N2 virus was identified on 26 June 2005 in Ibaraki Prefecture, Japan. As of April 2006, 41 layer farms have been identified as infected and approximately 3.4 million chickens have been destroyed. Since the infected chickens did not show any clinical signs or lesions, a serological surveillance program was launched to determine if any other farms were infected. All farms having an epidemiological relationship with the infected farms or that were located in the movement control area (a $5 \mathrm{~km}$ radius) were subjected to serological surveillance. In this surveillance, we used the agar gel precipitation (AGP) test for detection of type A influenza virus antibodies and the hemagglutination inhibition (HI) test for detection of antibodies to the HA antigen of $\mathrm{A} /$ chicken/Ibaraki/1/05 (H5N2), which was isolated at the first detected farm.

In instances where emergency sero-surveillance is required to detect infected farms, the efficacy and performance of serological tests can have a marked influence on the ability to detect farms that are infected [12]. In general, both the sensitivity (Se) and specificity ( $\mathrm{Sp}$ ) of the tests are estimated by comparison with an assumed perfect reference test (a gold standard), preferably using experimentally infected animals. However, at the time of implementation of this emergency sero-surveillance, there was not enough time to plan and conduct inoculation studies for the purpose of test evaluation. We therefore applied the Markov Chain Monte Carlo (MCMC) method using Gibbs sampling [6] to estimate test performance using data obtained from the ongoing AI surveillance. The MCMC method is one of the latent class (Bayesian) approaches that can be applied to obtain expected values of unknown parameters using observed data, and this approach has recently been applied to the estimation of Se and $\mathrm{Sp}$ for diagnostic tests without a gold standard $[1,4,5,7]$. We evaluated the Se and Sp of the
AGP and HI tests, which are currently used for AI surveillance.

In the serological surveillance for AI, serum samples were taken from at least 10 chickens selected randomly on farms in accordance with the contingency plan for AI in Japan [8]. Under this surveillance scheme, 114 serum samples were obtained from six neighboring farms (each farm kept between 10,000 and 40,000 chickens) on 25 June 2006, which was during the period when the first outbreak of AI was suspected (Table 1). The test results from these 114 samples were used for evaluation of the test performances by the MCMC method.

Table 1. Test results of the AGP and HI tests for six farms in Ibaraki Prefecture

\begin{tabular}{|c|c|c|c|c|c|}
\hline \multicolumn{3}{|c|}{ Farm ID } & \multirow{2}{*}{$\begin{array}{c}\mathrm{HI}^{\mathrm{b}}+ \\
32\end{array}$} & \multirow{2}{*}{$\begin{array}{c}\mathrm{HI}- \\
0\end{array}$} & \multirow{2}{*}{$\begin{array}{c}\text { Total } \\
32\end{array}$} \\
\hline 1 & $\left.\mathrm{AGP}^{\mathrm{a}}\right)_{+}$ & & & & \\
\hline & AGP- & \multirow{4}{*}{ Total } & 8 & 0 & 8 \\
\hline & & & 40 & 0 & 40 \\
\hline \multirow[t]{3}{*}{2} & AGP+ & & 8 & 0 & 8 \\
\hline & AGP- & & 12 & 0 & 12 \\
\hline & & \multirow[t]{3}{*}{ Total } & 20 & 0 & 20 \\
\hline \multirow[t]{3}{*}{3} & AGP+ & & 2 & 0 & 2 \\
\hline & $\mathrm{AGP}_{-}$ & & 0 & 8 & 8 \\
\hline & & \multirow[t]{3}{*}{ Total } & 2 & 8 & 10 \\
\hline \multirow[t]{3}{*}{4} & AGP+ & & 7 & 0 & 7 \\
\hline & $\mathrm{AGP}_{-}$ & & 5 & 0 & 5 \\
\hline & & Total & 12 & 0 & 12 \\
\hline \multirow[t]{3}{*}{5} & $\mathrm{AGP}+$ & & 5 & 0 & 5 \\
\hline & AGP- & & 1 & 6 & 7 \\
\hline & & Total & 6 & 6 & 12 \\
\hline \multirow[t]{3}{*}{6} & $\mathrm{AGP}+$ & & 10 & 0 & 10 \\
\hline & AGP- & & 6 & 4 & 10 \\
\hline & & Total & 16 & 4 & 20 \\
\hline
\end{tabular}

a) Agar gel immunodiffusion test with $48 \mathrm{hr}$ incubation.

b) Hemagglutination inhibition test with a cut-off value of $16 \mathrm{HI}$ titre. 
The AGP test was conducted in a diagnostic laboratory in Ibaraki Prefecture, and the AGP antigen was prepared from chorioallantoic membranes from eggs infected with A/budgerigar/Aichi/1/77 (H3N8) at the National Institute of Animal Health (NIAH). Preparation of the AGP antigen, virus inactivation by beta-propiolactone, and the test method were performed as described in the OIE Manual [9]. The antigen was placed into a center well, and test serum was added to an outer well with the positive reference sera on either side. Serum samples with which had precipitation line connected to that of reference positive serum were considered to be positive after $48 \mathrm{hr}$ incubation at room temperature.

The HI test was performed at NIAH with V-bottomed, 96-well microtitre plates as described in the OIE Manual [9]. The A/chicken/Ibaraki/1/05 (H5N2) isolated from the first farm in Ibaraki Prefecture where the disease was detected was inoculated into embryonated eggs, and the allantoic fluid was harvested. The virus contained in the allantoic fluid was then subjected to inactivation using formalin. Four HA units of the virus in $0.025 \mathrm{~m} l$ were mixed with diluted serum samples, incubated for $30 \mathrm{~min}$, and then incubated with $0.025 \mathrm{ml}$ of $0.5 \%(\mathrm{v} / \mathrm{v})$ chicken red blood cells for about $40 \mathrm{~min}$. The highest serum dilution showing complete inhibition of HA was expressed as an HI titer. HI titers of 16 or more were considered to be positive. The relationship between the two tests, farm by farm, is summarized in Table 1.

The test results for all 6 farms were evaluated simultaneously in order to estimate Ses and Sps for the two tests through application of the MCMC method. Assuming that the Ses and Sps of the two tests were the same on every farm and that the true prevalence at each farm was independent of the others, the expected values for row $i$, column $j$ of a $2 \times 2$ contingency table for the test results of farm $f\left(n_{f, i, j}\right)$ are described as follows:

$$
\begin{array}{r}
\mathrm{n}_{\mathrm{f}, 1,1}=\mathrm{P}_{\mathrm{f}} \mathrm{Se}_{1} \mathrm{Se}_{2}+\left(1-\mathrm{P}_{\mathrm{f}}\right)\left(1-\mathrm{Sp}_{1}\right)\left(1-\mathrm{Sp}_{2}\right) \\
\quad(\text { cells for AGP }+ \text { and } \mathrm{HI}+), \\
\mathrm{n}_{\mathrm{f}, 1,2}=\mathrm{P}_{\mathrm{f}} \mathrm{Se}_{1}\left(1-\mathrm{Se}_{2}\right)+\left(1-\mathrm{P}_{\mathrm{f}}\right)\left(1-\mathrm{Sp}_{1}\right) \mathrm{Sp}_{2} \\
\quad\left(\text { cells for AGP+ and } \mathrm{HI}^{-}\right), \\
\mathrm{n}_{\mathrm{f}, 2,1}=\mathrm{P}_{\mathrm{f}}\left(1-\mathrm{Se}_{1}\right) \mathrm{Se}_{2}+\left(1-\mathrm{P}_{\mathrm{f}}\right) \mathrm{Sp}_{1}\left(1-\mathrm{Sp}_{2}\right) \\
\quad(\text { cells for AGP- and HI+), } \\
\mathrm{n}_{\mathrm{f}, 2,2}=\mathrm{P}_{\mathrm{f}}\left(1-\mathrm{Se}_{1}\right)\left(1-\mathrm{Se}_{2}\right)+\left(1-\mathrm{P}_{\mathrm{f}}\right) \mathrm{Sp}_{1} \mathrm{Sp}_{2}, \\
\quad\left(\text { cells for AGP- and } \mathrm{HI}^{-}\right),
\end{array}
$$

where the true prevalence for farm $\mathrm{f}$ is $\mathrm{P}_{\mathrm{f}}$; the Ses of AGP and $\mathrm{HI}$ are $\mathrm{Se}_{1}$ and $\mathrm{Se}_{2}$, respectively; and the $\mathrm{Sps}$ of AGP and $\mathrm{HI}$ are $\mathrm{Sp}_{1}$ and $\mathrm{Sp}_{2}$, respectively. The conditional probability that the observed values were obtained for farm $f$ $\left(\mathrm{D}_{\mathrm{f}, \mathrm{i}, \mathrm{j}}\right)$ can be described using a multinomial distribution as follows:

$$
\begin{aligned}
& \left\{\mathrm{D}_{\mathrm{f}, 1,1}, \mathrm{D}_{\mathrm{f}, 1,2}, \mathrm{D}_{\mathrm{f}, 2,1}, \mathrm{D}_{\mathrm{f}, 2,2} \mid \mathrm{n}_{\mathrm{f}, 1,1}, \mathrm{n}_{\mathrm{f}, 1,2}, \mathrm{n}_{\mathrm{f}, 2,1}, \mathrm{n}_{\mathrm{f}, 2,2}, \mathrm{~T}_{\mathrm{f}}\right\} \\
& \sim \operatorname{multinomial}\left(\left(\mathrm{n}_{\mathrm{f}, 1,1}, \mathrm{n}_{\mathrm{f}, 1,2,}, \mathrm{n}_{\mathrm{f}, 2,1}, \mathrm{n}_{\mathrm{f}, 2,2}\right), \mathrm{T}_{\mathrm{f}}\right),
\end{aligned}
$$

where the total number of tested samples for farm $\mathrm{f}$ is $\mathrm{T}_{\mathrm{f}}$.

For Gibbs sampling, a non-informative prior distribution, Beta $(1,1)$, was used to estimate all unknown parameterse.g., $\mathrm{P}_{\mathrm{f}}, \mathrm{Se}_{1}, \mathrm{Se}_{2}, \mathrm{Sp}_{1}$, and $\mathrm{Sp}_{2}$. The details of the Gibbs sam-

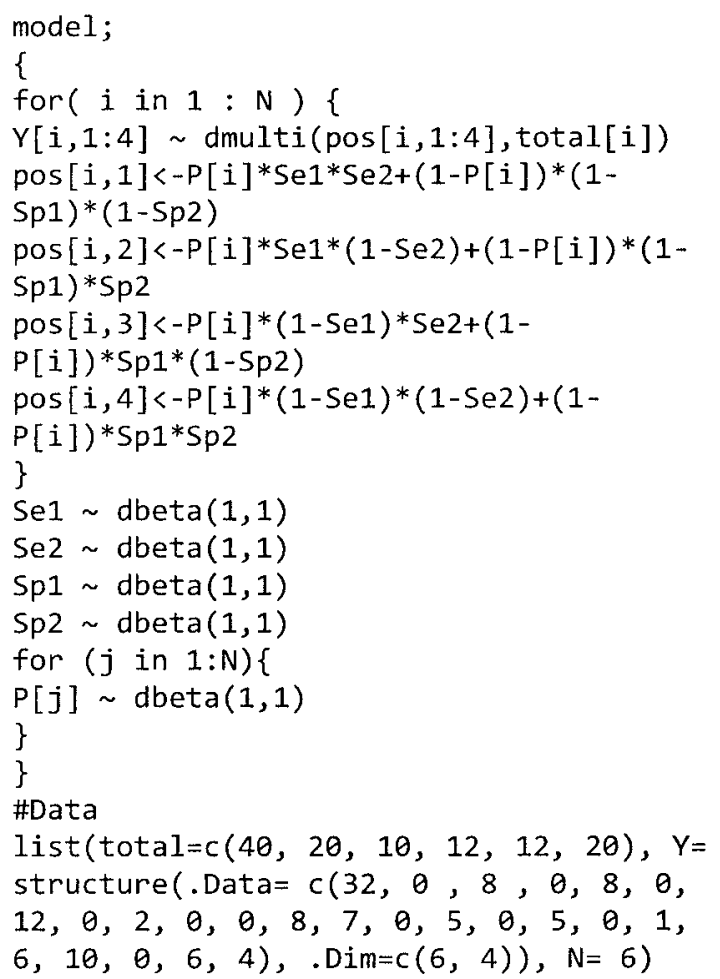

Fig. 1. Code of the model applied to BRugs.

Table 2. Median and 95\% credible interval (CI) of sensitivity (Se) and specificity (Sp) obtained by Gibbs sampling

\begin{tabular}{llcc}
\hline & & Median & $95 \% \mathrm{CI}^{\mathrm{c})}$ \\
\hline $\mathrm{AGP}^{\mathrm{a})}$ & $\mathrm{Se}$ & 0.674 & $0.574-0.772$ \\
& $\mathrm{Sp}$ & 0.958 & $0.796-0.999$ \\
$\mathrm{HI}^{\mathrm{b})}$ & $\mathrm{Se}$ & 0.992 & $0.957-1.000$ \\
& $\mathrm{Sp}$ & 0.899 & $0.593-0.996$
\end{tabular}

a) Agar gel precipitation test at $48 \mathrm{hr}$ incubation.

b) Hemagglutination inhibition test at a cut-off value of $16 \mathrm{HI}$ titre.

c) $95 \%$ credible interval.

pling method are described elsewhere [4, 5, 7]. A total of 20,000 iterations were performed to obtain posterior distributions from the last 19,500 iterations, thus removing the initial 500 iterations of the so-called "burn-in" period. Gibbs sampling was conducted using the statistical software $\mathrm{R}$ version 2.2.1 [10] with the BRugs package [11]. The details for the code applied to BRugs are shown in Fig. 1.

Table 2 shows median values with $95 \%$ credible intervals $(95 \% \mathrm{CI})$ for the estimated $\mathrm{Se}$ and $\mathrm{Sp}$ values when performance of the AGP test was judged after $48 \mathrm{hr}$ and a cut-off value of $16 \mathrm{HI}$ titre was used for the $\mathrm{HI}$ test. The Se of the AGP test $(0.67,95 \% \mathrm{CI}, 0.57-0.77)$ was lower than that of the HI test $(0.99,95 \% \mathrm{CI}, 0.96-1.00)$; however, the Sps were reasonably high for both the AGP test $(0.96,95 \% \mathrm{CI}, 0.80$ $1.00)$ and the HI test $(0.90,95 \% \mathrm{CI}, 0.59-1.00)$. The Se of 
the AGP test decreased to 0.40 when the AGP test results were judged after $24 \mathrm{hr}$, but the $\mathrm{Sp}$ remained high (0.95)(data not shown). This suggests that a 48-hr interval was optimal for the AGP test.

The HI test is commonly used as a gold standard to verify the performances of other diagnostic methods for AI, although the actual test performance of the HI test is not clear $[2,3,13]$. We calculated the Se and Sp of the AGP test from the test results for six farms using the results of the HI test as a gold standard in order to verify the MCMC method. The Se and Sp values of the AGP test were 0.67 (64/96) and $1.0(18 / 18)$, respectively. These results were consistent with the estimates from the MCMC method and suggested that the MCMC method could reproduce the relationship between the two test performances reasonably well.

Given the technical simplicity associated with the AGP test in addition to the non-subtype-specific affinity and an abundant antigen supply, its application for screening purposes can be justified for use in the early stages of outbreaks despite its relatively low Se. The HI test has strain-specific affinity and cannot detect other strains of AI virus. The HI test could be an alternative for primary screening due to its high Se, especially in high risk areas, if a specific strain of AI virus is believed to be the source of an outbreak, such as in the case of secondary infections, and if the necessary testing capacity is established, including a supply of antigen. In fact, the HI test and antigens derived from the virus isolated from the first case were used for primary screening of the movement control areas during later stages of this epidemic.

The MCMC method is useful for evaluating test performances within a relatively short time period in emergency situations. However, it should be noted that actual test performance may be influenced by the infection stage of the individual animals at the tested farms due to differences in the targeted antibodies in each test. Therefore, the length of time that has passed after disease introduction and before administration of the test may affect test performance on a farm-by-farm basis. As a result, it is recommended that test performance be monitored continuously during an epidemic.

We conclude that the MCMC method can potentially be a powerful tool for evaluation of diagnostic tests in the primary stage of emergency surveillance, even in instances where relatively little information is available on test performance.

\section{REFERENCES}

1. Branscum, A.J., Gardner, I.A. and Johnson, W.O. 2005. Prev. Vet. Med. 68: 145-163.

2. Capua, I., Cattoli, G. and Marangon, S. 2004. Dev. Biol. (Basel.) 119: 229-233.

3. Cattoli, G., Terregino, C., Brasola, V., Rodriguez, J.F. and Capua, I. 2003. Avian. Dis. 47: 1060-1062.

4. Enoe, C., Andersen, S., Sorensen, V. and Willeberg, P. 2001. Prev. Vet. Med. 51: 227-243.

5. Enoe, C., Georgiadis, M.P. and Johnson, W.O. 2000. Prev. Vet. Med. 45: 61-81.

6. Gelfand, A. and Smith, A.F.M. 1990. J. Am. Statist. Assoc. 85 : 398-409.

7. Joseph, L., Gyorkos, T.W. and Coupal, L. 1995. Am. J. Epidemiol. 141: 263-272.

8. MAFF. 2004. http://www.maff.go.jp/tori/bousi.htm (in Japanese.)

9. OIE. 2005. http://www.oie.int/eng/normes/mmanual/A_00037.htm

10. R Development Core Team. 2005. http://www.R-project.org

11. Thomas, A. 2006. http://mathstat.helsinki.fi/openbugs/

12. Tsutsui, T., Minami, N., Koiwai, M., Hamaoka, T., Yamane, I. and Shimura, K. 2003. Prev. Vet. Med. 61: 45-58.

13. Zhou, E.M., Chan, M., Heckert, R.A., Riva, J. and Cantin, M.F. 1998. Avian. Dis. 42: 517-522. 